\title{
On the Independence Number of Minimum Distance Graphs*
}

\author{
G. Csizmadia \\ Courant Institute, New York University, \\ New York, NY 10012, USA
}

\begin{abstract}
Let $F=F(n)$ denote the largest number such that any set of $n$ points in the plane with minimum distance 1 has at least $F$ elements, no two of which are at unit distance. Improving a result by Pollack, we show that $F(n) \geq \frac{9}{35} n$. We also give an $O(n \log n)$ time algorithm for selecting at least $\frac{9}{35} n$ elements in a set of $n$ points with minimum distance 1 so that no two selected points are at distance 1 .
\end{abstract}

\section{Introduction}

Let $X$ be a set of $n$ points in the plane with minimum distance 1 . The minimum distance graph associated with $X$ is a graph with vertex set $X$, in which two vertices are connected by an edge if and only if their distance is 1 .

Let $G$ be a graph with $n$ vertices. A subset $I$ of the vertex set of $G$ is said to be independent, if no two elements of $I$ are connected by an edge. The independence number $\alpha(G)$ of $G$ is defined as the size of the largest independent set of vertices in $G$. Let

$$
F(n)=\min \alpha(G),
$$

where the minimum is taken over all minimum distance graphs on $n$ vertices.

In 1983, Erdôs asked for bounds on $F(n)$. The vertex set of $\lfloor n / 3\rfloor$ widely spaced unit triangles shows that $F(n) \leq\left\lceil\frac{1}{3} n\right\rceil$. In 1996, Pach and Tóth [3] gave a better construction which yields $F(n) \leq\left\lceil\frac{5}{16} n\right\rceil$ for large $n$.

In 1985 , Pollack [4] proved $F(n) \geq\left\lceil\frac{1}{4} n\right\rceil$ by observing that the minimum distance graph is planar, therefore 4-colorable. Hence, the largest color class is an independent set of size at least $\frac{1}{4} n$. For planar graphs, in general, the bound $\alpha(G) \geq \frac{1}{4} n$ cannot be improved. However, for minimum distance graphs, we can establish a better bound.

\footnotetext{
* This research was supported by NSF Grant CCR-94-24398, OTKA-T-020914, and OTKA-14220.
} 
Theorem. Given $n$ points in the plane with minimum distance 1 , we can always choose at least $\frac{9}{35} n$ of them so that their minimum distance is greater than 1 .

Using the above terminology, the theorem states that $F(n) \geq \frac{9}{35} n$.

\section{Proof of the Theorem}

Let $X$ be a set of $n$ points in the plane with minimum distance 1. Let $G=G(X)$ denote the minimum distance graph associated with $X$. We will prove that $\alpha(G) \geq \frac{9}{35} n$. It is sufficient to prove the following lemma:

Lemma 1. Every minimum distance graph $G$ has an independent set $P$ of $k \leq 9$ vertices such that if $m$ is the number of vertices in $G-P$, incident to at least one element of $P$, then $k /(k+m) \geq \frac{9}{35}$.

The main theorem follows from Lemma 1 , since we can repeatedly apply the lemma to remove an independent set and its neighbors with the given property. The union of these independent sets is an independent set with at least $\frac{9}{35} n$ elements.

The proof uses two auxiliary results (Lemmas 2 and 3), whose proofs are postponed to the last section.

The main idea of the proof is the following. First we can suppose that all vertices have degree at least 3 . Then if you walk counterclockwise around the boundary of $G$, then the direction you are facing must rotate counter-clockwise around $360^{\circ}$. Only vertices of degree 3 contribute to this counter-clockwise rotation so the boundary must contain some such vertices. Moreover, the counterclockwise rotations at degree 3 vertices must outweigh the clockwise rotations at degree 4 and 5 vertices. A careful case analysis of the possible positions of the vertices and their neighbors gives the desired independent set $P$.

Proof of Lemma 1. We can make the following simple observations:

(a) If a vertex has degree 2 , then it forms an independent set satisfying the conditions of Lemma 1;

(b) if two nonadjacent vertices of degree 3 share a neighbor, then they form such an independent set; and

(c) if a vertex of degree 3 shares two neighbors with a nonadjacent vertex of degree 4 , then they form such a set; etc.

Let $d(p)$ denote the degree of a vertex $p$ of $G$. By the above observations, we can suppose that $d(p) \geq 3$ for all $p \in G$.

Define the boundary graph $C^{\prime}$ as follows. Let $r_{1}$ denote a vertex of $G$ with minimum $y$-coordinate. Draw a half-line from $r_{1}$ pointing downward, and turn it counterclockwise around $r_{1}$ until it hits a neighbor of $r_{1}$. Call that neighbor $r_{2}$. Then turn the segment $r_{1} r_{2}$ counterclockwise around $r_{2}$ until it hits another neighbor of $r_{2}$. Call that neighbor $r_{3}$. Continue this procedure until we hit a point $r_{i}$ that has been encountered before. Then $r_{i}=r_{i+t}$, so we have a cycle $C^{\prime}=\left\{r_{i+1}, r_{i+2}, \ldots, r_{i+t}\right\}$. 


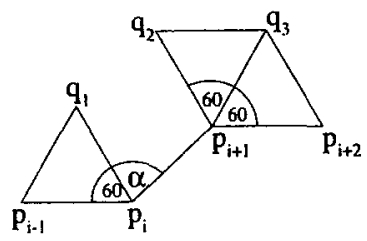

Fig. 1. Irregular $p_{i}$.

Let $C$ denote the path obtained from $C^{\prime}$ by the removal of $r_{i+t}$. For simplicity, denote the vertices of $C$ (in order) by $p_{1}, \ldots, p_{t-1}$. In the sequel $d(p)$ will denote the degree of $p$ in the original graph $G$.

Clearly, $3 \leq d\left(p_{i}\right) \leq 5$ for all $p_{i} \in C$. Let $a\left(p_{i}\right):=180^{\circ}-\angle p_{i-1} p_{i} p_{i+1}$ (the angle is understood in clockwise direction, facing the inner part of the cycle). Then $\sum_{i=1}^{t-1} a\left(p_{i}\right) \geq 240^{\circ}\left(\right.$ since $\left.a\left(p_{t}\right) \leq 120^{\circ}\right)$ and $a\left(p_{i}\right) \leq 240^{\circ}-d\left(p_{i}\right) \cdot 60^{\circ}$.

Definition. Let $p_{i-1}, q_{1}$, and $p_{i+1}$ denote the neighbors of a point $p_{i} \in C^{\prime}$ with $d\left(p_{i}\right)=3$ (in clockwise order as is shown in Fig. 1). If $\angle p_{i-1} p_{i} q_{1}=\angle q_{1} p_{i} p_{i+1}=60^{\circ}$, then $p_{i}$ is called regular. A vertex of degree 3 is called irregular if it is not regular.

If $p_{i}$ is irregular, then at least one of its neighbors, say $p_{i+1}$, does not have a common neighbor with $p_{i}$. If $d\left(p_{i+1}\right) \geq 4$, then call the $\left(p_{i}, p_{i+1}\right)$ pair a special pair. Note that an irregular $p_{i}$ may be the member of two special pairs.

Lemma 2. Let $p_{i} \in C$ be irregular, $d\left(p_{i+1}\right) \geq 4$, and $\angle q_{1} p_{i} p_{i+1}=\alpha>60^{\circ}$. Then $a\left(p_{i}\right)+a\left(p_{i+1}\right) \leq 0$ (see Fig. 1).

Thus, counterclockwise (positive) turns at irregular vertices are followed by clockwise (negative) turns of at least the same degree, unless the irregular vertex is followed by another vertex of degree 3 . In other words, for special pairs $\left(p_{i}, p_{i+1}\right)$ we have $a\left(p_{i}\right)+$ $a\left(p_{i+1}\right) \leq 0$.

Corollary. If $p_{i}$ is irregular and $\sum_{k=i-1}^{i+1} a\left(p_{k}\right)>0$, then $d\left(p_{i-1}\right)=3 \operatorname{ord}\left(p_{i+1}\right)=3$. Furthermore, if $\sum_{k=i-1}^{i+1} a\left(p_{k}\right)>60^{\circ}$, then $d\left(p_{i-1}\right)=d\left(p_{i+1}\right)=3$.

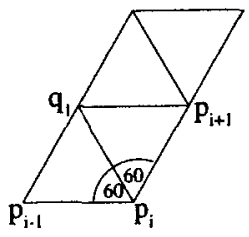

Fig. 2. Regular $p_{i}$. 
We may suppose that there are no two vertices $p_{j}, p_{j+2} \in C$ with $d\left(p_{j}\right)=d\left(p_{j+2}\right)=$ 3 , for otherwise $p_{j}$ and $p_{j+2}$ would have five neighbors altogether, and Lemma 1 would follow.

Next we choose a family of pairwise disjoint blocks (strings of consecutive vertices) in $C$. This procedure is the heart of the proof.

Going through the vertices of a negative block in counterclockwise direction yields a negative turn of at least $-60^{\circ}$, and all positive turns take place in a positive block. Therefore, since going around the boundary is a $360^{\circ}$ turn, there must be more positive blocks than negative ones.

(i) (a) Assume that a regular vertex $r_{i}$ has a neighbor of degree 5. Then fix such a neighbor $s_{i}$, and let $\left\{r_{i}, s_{i}\right\}$ be called a neutral block. (Note that no two neutral blocks have nonempty intersection, because $s_{i} \neq s_{j}$ unless $r_{i}=r_{j}$.)

(b) Assume that an irregular vertex $r_{i}$ is an element of a special pair. For each such $r_{i}$ fix an $s_{i}$ such that $\left(r_{i}, s_{i}\right)$ forms a special pair. Then $\left\{r_{i}, s_{i}\right\}$ is called a neutral block.

(ii) (a) If a regular vertex $r_{i}$ has no neighbor $s_{i}$ with $d\left(s_{i}\right)=5$, then it forms a positive block.

(b) Each pair of irregular vertices $\left(p_{i}, p_{i+1}\right)$ forms a positive block.

(iii) (a) Each $s_{i}$ with $d\left(s_{i}\right)=5$, which does not belong to any neutral block, forms a negative block.

(b) Each set of $l$ consecutive vertices $p_{k+1}, \ldots, p_{k+l}$, is called a negative block, whenever $d\left(p_{i}\right)=4(k+1 \leq i \leq k+l), d\left(p_{k}\right) \neq 4, d\left(p_{k+l+1}\right) \neq 4$, $\sum_{k+1}^{k+l} a\left(p_{i}\right) \leq-60^{\circ}$, and neither $p_{k+1}$ nor $p_{k+l}$ is a member of a neutral block.

Note that some vertices $p_{i_{1}}, \ldots, p_{i_{1}}$ may not belong to any of the blocks defined above, but for them $\sum_{j=1}^{l} a\left(p_{i_{j}}\right) \leq 0$ holds.

Clearly, each negative block contributes $-60^{\circ}$ (or less) to $\sum_{p_{i} \in C} a\left(p_{i}\right)$. Each positive turn not neutralized by the negative turn, at a following vertex of degree 4 or degree 5 , is now represented by a positive block. Moreover, by the corollary, a positive block contributes at most $60^{\circ}$ to $\sum_{p_{i} \in C} a\left(p_{i}\right)$. So $\sum_{p_{i} \in C} a\left(p_{i}\right) \geq 240^{\circ}$ implies

$$
\mid\{P: P \text { is a positive block }\}|\geq|\{N: N \text { is a negative block }\} \mid+4 \text {. }
$$

Let $B_{1}, \ldots, B_{s}$ denote the disjoint blocks in the order as they appear in $C$. There exists a positive block $\boldsymbol{B}_{j}$ for which $\boldsymbol{B}_{j+1}$ is either positive or neutral.

(i) If $B_{j}$ consists of a single regular vertex, then suppose it is $p_{2}$. It follows that $d\left(p_{1}\right) \leq 4$. If $B_{j}$ consists of two irregular vertices, then denote them by $p_{1}$ and $p_{2}$.

(ii) If $B_{j+1}$ is a positive block, and it is a regular vertex, then we can suppose that it is $p_{m}$. If $B_{j+1}$ consists of two irregular vertices, then denote them by $p_{m}$ and $p_{m+1}$.

(iii) If $B_{j+1}$ is a neutral block, then let $p_{m} \in B_{j+1}$ be the vertex in $B_{j+1}$ with degree 3 . In this case, either $d\left(p_{m-1}\right) \geq 4$ or $d\left(p_{m+1}\right) \geq 4$. 
Thus there are eight possible types of $\left(B_{j}, B_{j+1}\right)$ pairs, but in any case, the degrees of $p_{1}, p_{2}, \ldots, p_{m}$ are either:

(a) $4,3,3$; or

(b) $4,3, \leq 4, \leq 4, \ldots, \leq 4$, $\leq 5,3$; or

(c) $3,3, \leq 4, \leq 4, \ldots, \leq 4, \leq 5,3$;

and in cases (a) and (b), $p_{2}$ is regular and so the first and third vertices share two neighbors. $\leq 4$ and $\leq 5$ are denoting vertices of degree at most 4 and at most 5 , respectively.

This is all we need for the rest of the proof. We distinguish two cases:

(a) Suppose that there exists an $h$ with $3 \leq h \leq 15, h \leq m$, such that $d\left(p_{h}\right)=3$. There are no negative blocks between $B_{j}$ and $B_{j+1}$, therefore $d\left(p_{i}\right) \leq 4$ for all $i$ where $3 \leq i \leq m-2$. Then:

(i) if $h$ is even, set $P=\left\{p_{h}, p_{h-2}, \ldots, p_{2}\right\}$;

(ii) if $h$ is odd, set $P=\left\{p_{h}, p_{h-2}, \ldots, p_{1}\right\}$; and Lemma 1 follows.

(b) Let $m \geq 16$ and $d\left(p_{i}\right)=4(3 \leq i \leq 14)$. There was no negative block between $B_{j}$ and $B_{j+1}$, therefore $\sum_{3}^{14} a\left(p_{i}\right)>-60^{\circ}$.

Lemma 3. Suppose that there is a sequence $p_{1}, p_{2}, \ldots, p_{14}$ in $C$ such that $d\left(p_{2}\right)=3$, $d\left(p_{i}\right)=4(3 \leq i \leq 14), \sum_{3}^{14} a\left(p_{i}\right)>-60^{\circ}$, and $p_{1}$ and $p_{3}$ have at most six neighbors altogether.

Then we can find $k \leq 9$ independent vertices of $G$ such that the number of vertices incident to at least one of them is at most $3 k-1$.

Thus, Lemma 1 follows.

\section{Proofs of the Lemmas}

Proof of Lemma 2. We may suppose that $d\left(p_{i+1}\right)=4$. Denote the neighbors of $p_{i+1}$ by $p_{i}, q_{2}, q_{3}$, and $p_{i+2}$ (see Fig. 1). Clearly, $\angle q_{1} p_{i} p_{i+1}=\alpha$ implies $\angle p_{i} p_{i+1} q_{2} \geq 180^{\circ}-\alpha$. Therefore,

$$
\begin{aligned}
180^{\circ}-a\left(p_{i}\right)+180^{\circ}-a\left(p_{i+1}\right) & =\angle p_{i-1} p_{i} p_{i+1}+\angle p_{i} p_{i+1} p_{i+2} \\
& \geq 60^{\circ}+\alpha+\left(180^{\circ}-\alpha\right)+60^{\circ}+60^{\circ}=360^{\circ} .
\end{aligned}
$$

Hence $a\left(p_{i}\right)+a\left(p_{i+1}\right) \leq 0$.

For the proof of Lemma 3, we need the following claim.

Claim. Let $p_{0}, \ldots, p_{6}$ be consecutive vertices of $C$ such that $d\left(p_{i}\right)=4(1 \leq i \leq 5)$ and $a\left(p_{i}\right)+a\left(p_{i+1}\right)>-60^{\circ}(1 \leq i \leq 4)$. Then:

(i) $p_{k}$ and $p_{k+1}(k=1,2,3,4)$ have a common neighbor $r_{k}$ (see Fig. 3). 


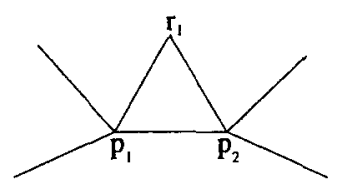

Fig. 3

Let $r_{0}$ and $r_{5}$ denote the remaining unlabeled neighbors of $p_{1}$ and $p_{5}$, respectively. Then:

(ii) $r_{k}(k=1,2,3,4)$ have at most two neighbors $\left(s_{k-1}, s_{k}^{\prime}\right)$ different from $p_{k}, p_{k+1}$, $r_{k-1}$, and $r_{k+1}$.

Suppose that both $r_{2}$ and $r_{3}$ have exactly two such neighbors and $\sum_{2}^{4} a\left(p_{i}\right)>-30^{\circ}$. Then:

(iii) $r_{2}$ and $r_{3}$ have a common neighbor $s_{2}=s_{2}^{\prime}\left(s_{2} \neq p_{3}\right)$; and

(iv) $s_{2}$ has at most two neighbors different from $s_{1}, r_{2}, r_{3}$, and $s_{3}^{\prime}$ (see Fig. 7).

Proof. (i) Let $k=1$ and suppose for contradiction that $p_{1}$ and $p_{2}$ have no common neighbor. Let us use the notation of Fig. 4. Clearly, $\angle b p_{1} p_{2}+\angle p_{1} p_{2} c \geq 180^{\circ}, \angle p_{0} p_{1} b \geq$ $120^{\circ}$, and $\angle c p_{2} p_{3} \geq 120^{\circ}$. So

$$
\begin{aligned}
a\left(p_{1}\right)+a\left(p_{2}\right) & =180^{\circ}-\angle p_{0} p_{1} p_{2}+180^{\circ}-\angle p_{1} p_{2} p_{3} \\
& \leq 360^{\circ}-180^{\circ}-120^{\circ}-120^{\circ}=-60^{\circ} .
\end{aligned}
$$

This is a contradiction, so Claim (i) is true for $k=1$. For $k=2,3,4$, the proof is similar.

(ii) Suppose $k=1$ and that $p_{1}$ and $p_{2}$ have the common neighbor $r_{1}$, and $r_{1}$ has three neighbors $s_{1}, s_{2}, s_{3}$ as is shown in Fig. 5 .

Let $\alpha=r_{0} p_{1} r_{1}$ and $\beta=r_{1} p_{2} r_{2}$. Then $\alpha+\beta<180^{\circ}$. We also know that $\angle p_{1} r_{1} s_{1} \geq$ $180^{\circ}-\alpha, \angle s_{3} r_{1} p_{2} \geq 180^{\circ}-\beta$. But

$$
\begin{aligned}
360^{\circ} & =\angle p_{1} r_{1} s_{1}+\angle s_{1} r_{1} s_{2}+\angle s_{2} r_{1} s_{3}+\angle s_{3} r_{1} p_{2}+\angle p_{2} r_{1} p_{1} \\
& \geq\left(180^{\circ}-\alpha\right)+60^{\circ}+60^{\circ}+\left(180^{\circ}-\beta\right)+60^{\circ}=540^{\circ}-(\alpha+\beta)>360^{\circ},
\end{aligned}
$$

a contradiction. For $k=2,3,4$, the proof is similar.

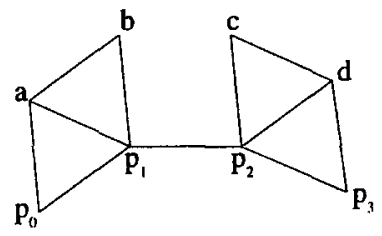

Fig. 4 


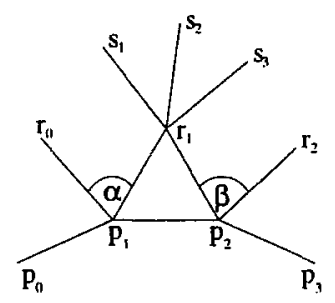

Fig. 5

(iii) Suppose that $s_{2} \neq s_{2}^{\prime}$ (see Fig. 6). Let $\left|r_{2} r_{3}\right|=t$ and $\left|s_{2}^{\prime} s_{2}\right|=s$. Let $\alpha=\angle r_{1} p_{2} r_{2}$, $\beta=\angle r_{2} p_{3} r_{3}$, and $\gamma=\angle r_{3} p_{4} r_{4}$. Then $\alpha+\beta+\gamma<210^{\circ}$. Clearly, $t=2 \sin (\beta / 2)$ and

$$
\begin{aligned}
\delta & =\angle s_{2}^{\prime} r_{2} r_{3} \leq 360^{\circ}-\left(\angle p_{2} r_{2} s_{1}+\angle s_{1} r_{2} s_{2}^{\prime}+\angle r_{3} r_{2} p_{3}+\angle p_{3} r_{2} p_{2}\right) \\
& \leq 360^{\circ}-\left[\left(180^{\circ}-\alpha\right)+60^{\circ}+\left(90^{\circ}-\frac{\beta}{2}\right)+60^{\circ}\right]=\alpha+\frac{\beta}{2}-30^{\circ} \\
& <210^{\circ}-\gamma-\frac{\beta}{2}-30^{\circ}=180^{\circ}-\gamma-\frac{\beta}{2} \leq 120^{\circ}-\frac{\beta}{2}
\end{aligned}
$$

Similarly, $\delta^{\prime}=\angle r_{2} r_{3} s_{2}<120^{\circ}-\beta / 2$. This implies

$$
\frac{t-s}{2} \geq \sin \left(90^{\circ}-\max \left\{\delta, \delta^{\prime}\right\}\right)>\sin \left[90^{\circ}-\left(120^{\circ}-\frac{\beta}{2}\right)\right]=\sin \left(\frac{\beta}{2}-30^{\circ}\right) \text {. }
$$

So

$$
s<t-2 \sin \left(\frac{\beta}{2}-30^{\circ}\right)=2\left[\sin \frac{\beta}{2}-\sin \left(\frac{\beta}{2}-30^{\circ}\right)\right] \leq 1,
$$

because $30^{\circ} \leq \beta / 2<45^{\circ}$. This is a contradiction, so $s_{2}=s_{2}^{\prime}$.

(iv) In part (iii), we proved $s_{2}=s_{2}^{\prime}$. Let $\alpha, \beta, \gamma$ be as before, and let $\varepsilon=\angle s_{1} r_{2} s_{2}$ and $\varepsilon^{\prime}=\angle s_{2} r_{3} s_{3}^{\prime}$. We get that $\varepsilon \leq \alpha+\beta-60^{\circ}$ and $\varepsilon^{\prime} \leq \gamma+\beta-60^{\circ}$ (see Fig. 7). We also

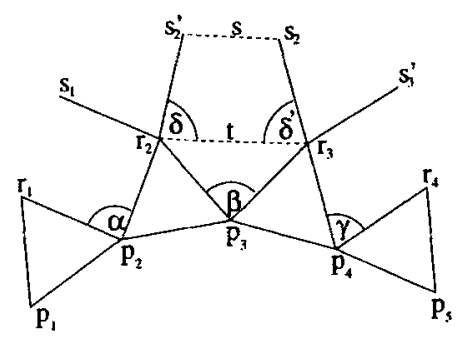

Fig. 6 


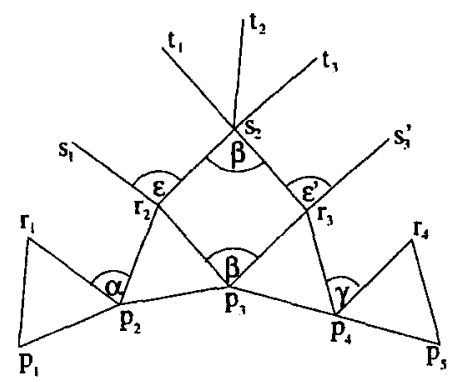

Fig. 7

know that $\angle r_{3} s_{2} r_{2}=\beta$. Thus if $s_{2}$ had three neighbors $t_{1}, t_{2}, t_{3}$, then we would get

$$
\begin{aligned}
360^{\circ} & \geq \beta+\left(180^{\circ}-\varepsilon\right)+60^{\circ}+60^{\circ}+\left(180^{\circ}-\varepsilon^{\prime}\right) \\
& \geq \beta+480^{\circ}-\left(\alpha+\beta-60^{\circ}\right)-\left(\beta+\gamma-60^{\circ}\right) \\
& =600^{\circ}-(\alpha+\beta+\gamma)>600^{\circ}-210^{\circ}=390^{\circ},
\end{aligned}
$$

which is a contradiction.

Now we are ready to prove Lemma 3.

Proof of Lemma 3. Claim (i) implies that all $p_{i}, p_{i+1}$ pairs $(3 \leq i \leq 13)$ have a common neighbor $r_{i}$ (see Fig. 8). Suppose that there is an $r_{j}(4 \leq j \leq 12)$ which has only one neighbor different from $r_{j-1}, r_{j+1}, p_{j}$, and $p_{j+1}$.

Now:

(a) if $j$ is odd, then pick $p_{j+2}, r_{j}, p_{j-1}, p_{j-3}, \ldots, p_{2}$; and

(b) if $j$ is even, then pick $p_{j+2}, r_{j}, p_{j-1}, p_{j-3}, \ldots, p_{1}$.

These $\lfloor j / 2\rfloor+2$ vertices have altogether $3(\lfloor j / 2\rfloor+2)-1$ neighbors.

Thus, we can suppose by Claim (ii) that all $r_{j}(4 \leq j \leq 12)$ has two other neighbors. $\sum_{3}^{14} a\left(p_{i}\right)>-60^{\circ}$ implies that either $\sum_{4}^{8} a\left(p_{i}\right)>-30^{\circ}$ or $\sum_{9}^{13} a\left(p_{i}\right)>-30^{\circ}$. Without loss of generality suppose $\sum_{9}^{13} a\left(p_{i}\right)>-30^{\circ}$.

Claim (iii) implies that there exists $s_{9}=s_{9}^{\prime}, s_{10}=s_{10}^{\prime}$, and $s_{11}=s_{11}^{\prime}$ (see Fig. 8). By Claim (iv), $s_{10}$ has at most two neighbors different from $s_{9}, s_{11}, r_{10}$, or $r_{11}$. Let

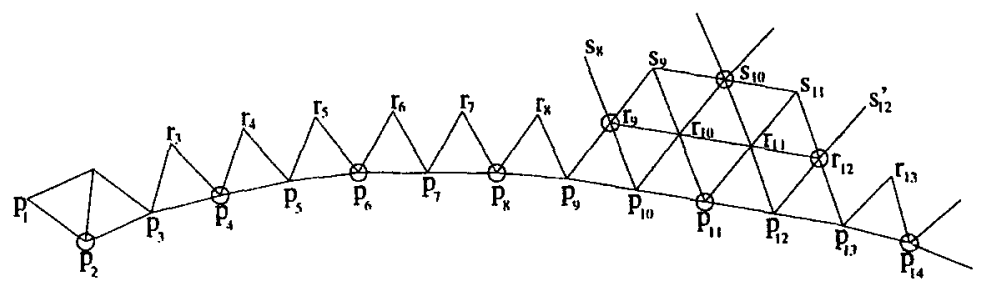

Fig. 8 
$P=\left\{s_{10}, r_{12}, r_{9}, p_{14}, p_{11}, p_{8}, p_{6}, p_{4}, p_{2}\right\}$. Then the number of points incident to at least one element of $P$ is at most $26=3 \cdot 9-1$, completing the proof of Lemma 3 .

Remark. The above proof gives us an algorithm for selecting at least $\frac{9}{35} n$ elements in a set of $n$ points with minimum distance 1 so that no two selected points are at distance 1. The algorithm finds at most 9 and at least 1 , new independent points in each step.

The minimum distance graph can be constructed in $O(n \log n)$ time, and after that, each step takes at most $C$ time, where $C$ is a large constant. We have to make at most $n$ steps, therefore the algorithm can be completed in $O(n \log n)$ time.

\section{References}

1. P. Erdôs. Some combinatorial and metric problems in geometry, In: Intuitive Geometry, Colloquia Mathematica Societatis János Bolyai, vol. 48 (K. Böröczky and G. Fejes Tóth, Eds.), North-Holland, Amsterdam, pp. $167-177,1985$.

2. J. Pach and P. K. Agarwal. Combinatorial Geometry, Wiley, New York, 1995.

3. J. Pach and G. Tóth. On the independence number of coin graphs, Geombinatorics 6(1), 30-33, 1996.

4. R. Pollack. Increasing the minimum distance of a set of points, J. Combinatorial Theory, Series A, 40, 450, 1985.

Received June 12, 1996, and in revised form April 22, 1997. 